\title{
The Effect of Probiotic Plus Prebiotic Supplementation on the Tolerance and Efficacy of Helicobacter Pylori Eradication Quadruple Therapy: a Randomized Prospective Double Blind Controlled Trial
}

\author{
Afshin Shafaghi ${ }^{1}$, Aydin Pourkazemi ${ }^{2 *}$, Mohsen Khosravani ${ }^{3}$, Saba Fakhrie Asl ${ }^{4}$, \\ Alireza Amir Maafi ${ }^{5}$, Zahra Atrkar Roshan ${ }^{5}$, Jafar Abaspour Rahimabad ${ }^{6}$
}

1. Member of Gastrointestinal and Liver Diseases Research Center(GLDRC) and Gastroenterology Department, Guilan University of Medical Sciences, Rasht, Iran gjhsdfgjfdhj

2. Infectious Disease Department, Guilan University of Medical Sciences, Rasht, Iran

3. Guilan University of Medical Sciences, Rasht, Iran

4. Gastroenterology Department, Guilan University of Medical Sciences, Rasht, Iran

5. Student Research Center, Guilan University of Medical Sciences, Rasht, Iran

6. Member of Caspian Tamin Pharmaceutical Company, Rasht, Iran

* Corresponding Author:

Aydin Pourkazemi, MD Razi Hospital, Sardar Jangal St., Rasht, Guilan, Iran

Postal code: 41448-95655

Telefax: + 981313228842

Email: dr.aydin.pourkazemi@gmail.com

Received: 26 Jan. 2016

Accepted: 01 Jun. 2016
ABSTRACT

\section{BACKGROUND}

Standard anti-Helicobacter pylori (H. pylori) treatment fails in the eradication of the organism in almost $10-35 \%$ of the patients and has different side effects. Recent studies have proposed that probiotic supplementations with or without prebiotic may improve the eradication rate and diminish the side effects, although it is still a controversial issue. We aimed to investigate the effect of probiotic with prebiotic supplementation on the eradication rate and side effects of anti $H$. pylori quadruple therapy.

\section{METHODS}

76 patients with a positive biopsy specimen for H. pylori were enrolled. They were randomized to receive quadruple therapy of bismuth, clarithromycin, amoxicillin, and omeprazole for 14 days and also the synbiotic or the placebo. We asked them to answer study questionnaires at the beginning and during the treatment. Finally, urea breath test was done 8 weeks after the treatment.

\section{RESULTS}

The eradication rate was significantly better in the synbiotic group by intention-to-treat analysis $(\mathrm{p}<0.05)$. Treatment side effects such as diarrhea, nausea, vomiting, epigastric pain, flatulence, constipation, and taste abnormality were similar in both groups but anorexia was significantly better in the synbiotic group $(\mathrm{p}<0.05)$.

\section{CONCLUSION}

The eradication rate was significantly better in the synbiotic group by intention-to-treat analysis $(\mathrm{p}<0.05)$. Treatment side effects such as diarrhea, nausea, vomiting, epigastric pain, flatulence, but could improve the eradication by augmenting the treatment tolerance and compliance.

\section{KEYWORDS:}

H. pylori eradication; Probiotic; Prebiotic; Synbiotic; Quadruple therapy; RCT

Please cite this paper as:

Shafaghi A, Pourkazemi A, Khosravani M, Fakhrie Asl S, Amir Maafi AR, Atrkar Roshan Z, Abaspour Rahimabad J. The Effect of Probiotic Plus Prebiotic Supplementation on the Tolerance and Efficacy of Helicobacter Pylori Eradication Quadruple Therapy: a Randomized Prospective Double Blind Controlled Trial. Middle East J Dig Dis 2016;8:179-188. DOI : 10.15171/mejdd.2016.30 


\section{INTRODUCTION}

Helicobacter pylori (H. Pylori), as a common and significant health problem plays a major contributory role in the pathogenesis of chronic gastritis, peptic ulcer disease, gastric mucosa associated lymphoid tissue (MALT) lymphoma, and adenocarcinoma. ${ }^{1-4}$ Currently, there are various therapeutic regimens; although the most successful one cannot eradicate the bacteria in at least $10 \%$ of the affected patients. ${ }^{5}$ Quadruple therapy is appropriate as initial therapy in areas where the prevalence of resistance to clarithromycin or metronidazole is $>20 \%$, or in patients with recent or repeated exposure to clarithromycin or metronidazole. ${ }^{6}$ A meta-analysis of 93 studies showed that in populations with either clarithromycin or metronidazole resistance, quadruple therapy resulted in a higher rate of eradication than triple therapy. ${ }^{7}$ Beside the eradication rate of treatment regimen, undesirable side effects, and patients' poor compliance play considerable role in the outcome of treatment. A considerable number of patients experience undesirable side effects. The most common complaints during antimicrobial treatments are diarrhea, epigastric pain, nausea, and bloating. ${ }^{8}$

Probiotic bacteria, defined as living microorganisms that have beneficial health effects to the host, have been successfully used in the treatment or prevention of several gastrointestinal (GI) disorders, such as antibioticassociated diarrhea, inflammatory bowel disease, and irritable bowel syndrome. ${ }^{9-10}$ They may be useful adjuncts to improve tolerability and compliance of anti $H$. pylori treatment. ${ }^{11-12}$ Because some probiotics have antimicrobial effects, they have been proposed as a treatment option for H. pylori infection but they should not be considered as a substitute for standard antibiotic treatments. ${ }^{13}$ Several studies have reported that certain probiotic bacteria, such as lactobacillus spp., exhibit inhibitory activity against H. pylori in vitro and in vivo. ${ }^{14-15}$

This study was designed to investigate the effect of probiotic-prebiotic supplementation on the eradication rate of anti $H$. pylori quadruple therapy, treatment side effects, and the improvement of dyspepsia after treatment. We aimed to investigate the effect of this supplementation, which contains Lactobacillus casei, Lactobacillus rhamnosus, Streptococcus thermophilus, Bifidobacterium breve, Lactobacillus acidophilus, Bifidobacterium longum, and Lactobacillus bulgarius on the eradication rate of anti $H$. pylori quadruple therapy, its side effects, and patients' compliance.

\section{MATERIALS AND METHODS}

\section{Study design}

This study was a prospective randomized double blind placebo controlled study. 76 patients with a positive biopsy specimen for $H$. pylori infection were enrolled from May 2011 to March 2012 in Rasht and Anzali, Iran (male/ female: 35/41, mean age: $43.5 \pm 13.31$ ). The patients who received 14 days of quadruple therapy consisted of bismuth subcitrate four times a day, and amoxicillin $1 \mathrm{~g}$, clarithromycin $500 \mathrm{mg}$, and omeprazole $20 \mathrm{mg}$, all twice a day were randomly assigned to receive synbiotic (Protexin Balance ${ }^{\circledR}$ ) or placebo 2 capsules before the lunch during the treatment and also three days earlier. We asked them to begin the synbiotic (treatment group, $\mathrm{n}=38$ ) or placebo (control group, $\mathrm{n}=38$ ) three days before initiation of the main treatment to make a better chance for colonization of probiotic bacteria. The patients were divided into two groups by random allocation. The placebo was manufactured by Sobhan Daru Company and there was no difference in shape, color, weight, and taste between the two products. Boxes containing placebo or probiotic were identical in shape, size, and color and did not have any trade mark and contained the same number of drugs. All the patients were followed up for 4-8 weeks after the end of treatment and to evaluate the patients compliance, pill counting was done. 8 weeks after the end of treatment, the eradication was confirmed by urea breath test (figure 1)

The inclusion criterion was the presence of $H$. pylori in the histopathological examination of biopsy specimen obtained during upper GI endoscopy. The gastric body greater curvature and prepyloric antrum were the preferred sites of biopsy sampling and a large channel biopsy forceps was used. Both hematoxylin and eosin (H\&E) and Giemsa staining were used and all the samples were evaluated by an expert histopathologist. A rapid urease test was also performed for a better confirmation. The exclusion criteria were GI bleeding, malignancy, inflammatory bowel disease,prior gastrectomy, recent history of $H$. pylori eradication, immunodeficiency, and the use of antibiotic or probiotics in the weeks before and during the study. Dyspeptic symptoms were 


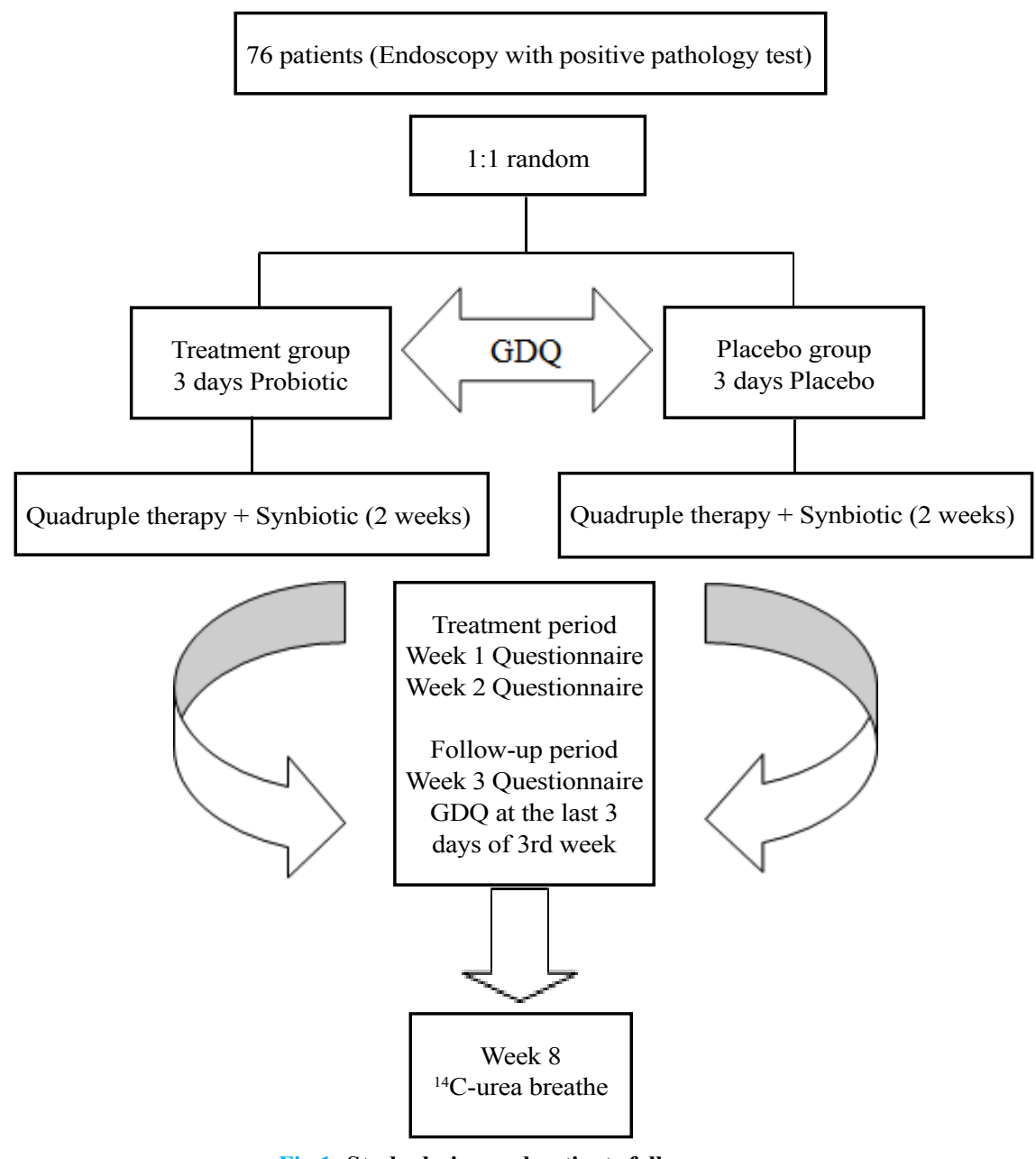

Fig.1: Study design and patients follow-up

recorded daily before the initiation of treatment in three days and also in the last three days of the third week after the initiation of treatment using modified version of the Glasgow Dyspepsia Questionnaire (GDQ). ${ }^{16}$ The dyspepsia score were ranged from 0-12. The assessment of side effects was done at the end of the 1st, 2 nd, and 3rd week after initiation of quadruple therapy by the modified version of the questionnaire by de Boer and colleagues. ${ }^{17}$ The subjects were asked to report any side effects during and after therapy such as taste disturbance, nausea, vomiting, epigastric pain, bloating, diarrhea, and constipation. They were also asked to grade each side effect according to severity as: mild (effect observed, but could be disregarded), moderate (effect sometimes interfered with daily activities), or severe (effect continuously interfered with daily activities). In order to obtain the highest compliance in registering any treatment related side effects, verbal explanations and printed instructions were both given to patients on how and when to fill in the questionnaires. This study was approved by the Ethics Committee of Guilan University of Medical Sciences and registered on the Iranian Registry of Clinical Trials website (IRCT registration no. IRCT201011135174N1). A written informed consent was obtained from each patient before the initiation of the study.

\section{H. pylori Eradication}

H. pylori eradication was evaluated by a ${ }^{14} \mathrm{C}$-urea breath test performed 8 weeks after the end of treatment. During this period the patients did not take any antibiotics or acid reducing drugs. After taking urea labeled with ${ }^{14} \mathrm{C}$ by the patients, $H$. pylori infection could be diagnosed by using Helitracer HUBT-20 (Headway Bio-Sci\&Tech Co, LTD, Shenzhen Zhonghe, China, sen- 
sitivity $95 \%$, specificity $95-100 \%$ ) to detect the volume of $\left[{ }^{14} \mathrm{C}\right]$ in exhaled breath due to action of urease generated by H.pylori that dissolve urea into ${ }^{14} \mathrm{CO} 2$. The entire test is finished in 25 minutes.

\section{Synbiotic Supplementation}

Protexin Balance provides a complex blend of 7 strains of friendly bacteria and prebiotic, to help maintain healthy digestive and immune systems in all the family packed in easy to swallow capsules. Protexin probiotics are Lactobacillus casei, Lactobacillus rhamnosus, Streptococcus thermophilus, Bifidobacterium breve, Lactobacillus acidophilus, Bifidobacterium longum, Lactobacillus bulgaricus, (TVC: 200 million CFU TVC: $2 \times 108$ CFU) and also FOS (fructooligosaccharide-prebiotic), magnesium stearate (source: mineral and vegetable), and vegetable capsule (hydroxypropyl methyl cellulose). These processes were accelerated by the oral administration of lactulose, a non-digestible disaccharide used as substrate by the Lactobacillus. Protexin is manufactured in stateof-the-art facility in Somerset, UK. (Probiotics International Ltd Somerset, TA13 5JH, UK Registered Company No: 1122942) \{available at http://www.protexin. com $\}$

\section{Statistical analysis}

Eradication rates were determined for both groups on a per-protocol (PP) and intention-to-treat (ITT) basis. For ITT analysis, all the enrolled patients were included, but for PP analysis, those who had taken less than $80 \%$ of any of the prescribed drugs, had dropped out due to severe adverse events, had not undergone final ${ }^{14} \mathrm{C}$-urea breath testing, or those who had violated the study protocol were excluded. The side effects, dyspepsia improvement, and the eradication rates were compared by paired Student $t$ test, Mann-Whitney U- test, Chi-square or Fischer's exact test. Results were considered significant when $p$ values were less than 0.05 and the analysis was done by SPSS software version 18 for windows.

\section{RESULTS}

\section{Patients/population}

76 patients (35 men, 41 women) were randomly allocated into placebo group (17 men, 21 women) and probiotic group (18 men, 20 women). In the placebo group, three patients could not complete the pre-treatment regimen i.e. the short course of placebo before the main therapy, and thereby were excluded from the study (one due to severe infectious diarrhea at the beginning of treatment, the other due to fascioliasis and hospital admission, and the last one due to the possibility of pregnancy). 11 patients in the placebo group were also excluded due to follow-up loss and non-compliance. Of the 14 excluded patients, 12 had non-ulcer dyspepsia (NUD) and 2 had duodenal ulcer (DU). In placebo group, of the 24 patients enrolled in PP analysis, 19 (79.16\%) had NUD and 5 (20.84\%) had DU with no case of gastric ulcer (GU). Out of 38 patients enrolled in ITT analysis, $31(81.57 \%)$ had NUD and $7(18.43 \%)$ had DU with no case of GU. There was no statistical difference in underlying diseases between the two groups (table 1). In the synbiotic group, all the 38 patients completed treatment regimen with favorable compliance and there was no missed case.

\section{H. pylori eradication rate}

The overall eradication rate in patients who completed the treatment regimen was $95.2 \%$ (CI 95\%: 89.9-100). $79 \%$ of these patients had NUD, which the eradication rate was $93.9 \%$ in this group of patient.

Based on ITT analysis, the eradication rates were 92.1\% (CI 95\%: 83.5-100\%) in the synbiotic group and $63.2 \%$ (CI 95\%: 47.9- 78.5\%) in the placebo group. The difference was statistically significant. However, there was no statistically difference between the two groups based on PP analysis (table 2).

\section{Adverse effects and dyspepsia improvement}

We did not find any statistically significant difference in frequency and severity of flatulence, taste abnormality, epigastric pain, nausea and vomiting, constipation, and diarrhea between the two groups but anorexia was significant in treatment period $(\mathrm{p}<0.05)$. The most common symptoms were taste abnormality and flatulence that were detected in most of the patients in treatment period (table 3 ).

The mean GDQ score before (3.36 \pm 3.25$)$ and after $(1.76 \pm 2.61)$ treatment in placebo group was statisti- 
Table 1: Comparison of patients' characteristic in study groups

\begin{tabular}{|c|c|c|c|}
\hline Variables & Probiotic $(n=38)$ & Placebo $(n=38)$ & P value \\
\hline Sex & & & NS \\
\hline Male & $18(47.36 \%)$ & $17(44.73 \%)$ & \\
\hline Female & $20(52.63 \%)$ & $21(55.27 \%)$ & \\
\hline Age (years) & & & NS \\
\hline Mean \pm SD & $43.7513 .32 \pm$ & $43.35 \pm 12.29$ & \\
\hline Weight (Kg) & & & $<0.05$ \\
\hline Mean \pm SD & $66.87 \pm 10.29$ & $74.35 \pm 16.07$ & \\
\hline Smoking & & & NS \\
\hline No & $30(78.94 \%)$ & $30(78.94 \%)$ & \\
\hline Ex & $5(13.15 \%)$ & $2(5.26 \%)$ & \\
\hline Active & $3(7.91)$ & $6(15.78)$ & \\
\hline Endoscopic findings & & & NS \\
\hline DU & $8(21.05 \%)$ & $7(18.42 \%)$ & \\
\hline GU & 0 & 0 & \\
\hline NUD & $30(78.95 \%)$ & $31(81.58 \%)$ & \\
\hline
\end{tabular}

Table 2: Eradication rate between two study groups based on method of analysis

\begin{tabular}{lccc}
\hline Variable & Probiotic group & Placebo group & P value \\
\hline $\begin{array}{l}\text { Eradication } \\
\text { ITT analysis }\end{array}$ & $92.1 \%(83.5-100 \%)$ & $63.15 \%(47.9-78.5 \%)$ & $\mathrm{P}<0.05$ \\
\hline \multicolumn{1}{c}{ PP analysis } & $92.1 \%(83.5-100 \%)$ & $100 \%(24$ of 24$)$ & $\mathrm{NS}$ \\
\hline NS= Not Significant & & &
\end{tabular}

cally different $(\mathrm{P}<0.05)$. The mean GDQ score before $(2.76 \pm 2.07)$ and after $(1.53 \pm 1.54)$ treatment in symbiotic group was statistically different $(\mathrm{P}<0.05)$ but there was no difference between GDQ scores of patients in the two groups before and after treatment, respectively (table 4).

\section{DISCUSSION}

H. pylori associated diseases that require effective eradication, has increased and we encounter some problems such as treatment resistance and failure. Patients frequently experience GI upset, diarrhea, nausea, vomiting, anorexia, and bloating associated with eradication. Drugs side effects can lead to discontinuation of therapy. The most important predictors of anti $H$. pylori treatment failure are poor compliance and antibiotic resistance.

Probiotics are defined by the FAO/WHO as live microorganisms, which are administered in adequate amounts, confer a health benefit on the host and are widely used as a live microbial food supplement that can improve the intestinal microbial balance. ${ }^{18}$ Prebiotics are currently defined as a non-digestible food ingredient that beneficially affects the host by selectively stimulating the growth and/or activity of one or a limited number of bacteria in the colon. ${ }^{19}$ Galactooligosaccharides are one category of prebiotics, which contain growth-promoting factors for Bifidobacterium. Synbiotics are generally considered as a combination of probiotics and prebiotics.

Shimizu and colleagues showed that Bifidobacterium breve strain Yakult and Lactobacillus casei strain Shirota as probiotics and galactooligosaccharides as prebiotics, may cause significantly greater levels of benefit in sepsis. Bifidobacterium and Lactobacillus and short chain fatty acids (SCFAs) cause lower incidence of infectious complications such as enteritis, pneumonia, and bacteremia than those who received no synbiotics. ${ }^{20}$ The presumptive mechanism of synbiotics is that their administration increases the levels of beneficial bacteria such as Bifidobacterium and Lactobacillus. The increased level of total anaerobes induces increased production of SCFAs in GI tract. These environmental changes can 
Table 3: Prevalence of the most frequent tolerable side effects occurred in patients of each groups and comparison of them

\begin{tabular}{|c|c|c|c|c|}
\hline Variables & & $\begin{array}{l}\text { Placebo } \\
\text { group }\end{array}$ & $\begin{array}{l}\text { Probiotic } \\
\text { group }\end{array}$ & p-value \\
\hline \multirow[t]{3}{*}{ Diarrhea } & $10^{\text {th }}$ day & $18.2 \%$ & $8.3 \%$ & NS \\
\hline & $17^{\text {th }}$ day & $10 \%$ & $8.3 \%$ & NS \\
\hline & $24^{\text {th }}$ day & 0 & $5.6 \%$ & NS \\
\hline \multirow[t]{3}{*}{ Epigastric pain } & $10^{\text {th }}$ day & $59.1 \%$ & $47.2 \%$ & NS \\
\hline & $17^{\text {th }}$ day & $40 \%$ & $36.1 \%$ & NS \\
\hline & $24^{\text {th }}$ day & $50 \%$ & $39.4 \%$ & NS \\
\hline \multirow[t]{3}{*}{ Flatulence } & $10^{\text {th }}$ day & $50 \%$ & $50 \%$ & NS \\
\hline & $17^{\text {th }}$ day & $45 \%$ & $45.7 \%$ & NS \\
\hline & $24^{\text {th }}$ day & $30 \%$ & $55.9 \%$ & NS \\
\hline \multirow[t]{3}{*}{ Taste abnormality } & $10^{\text {th }}$ day & $81.8 \%$ & $77.8 \%$ & NS \\
\hline & $17^{\text {th }}$ day & $75 \%$ & $61.1 \%$ & NS \\
\hline & $24^{\text {th }}$ day & $45 \%$ & $38.2 \%$ & NS \\
\hline \multirow[t]{3}{*}{ Constipation } & $10^{\text {th }}$ day & $40.9 \%$ & $33.3 \%$ & NS \\
\hline & $17^{\text {th }}$ day & $20 \%$ & $36.1 \%$ & NS \\
\hline & $24^{\text {th }}$ day & $15 \%$ & $33.3 \%$ & NS \\
\hline \multirow[t]{3}{*}{ Nausea } & $10^{\text {th }}$ day & $26.1 \%$ & $28.6 \%$ & NS \\
\hline & $17^{\text {th }}$ day & $33.3 \%$ & $36.1 \%$ & NS \\
\hline & $24^{\text {th }}$ day & $20 \%$ & $17.6 \%$ & NS \\
\hline \multirow[t]{3}{*}{ Vomiting } & $10^{\text {th }}$ day & $4.3 \%$ & $5.6 \%$ & NS \\
\hline & $17^{\text {th }}$ day & $4.8 \%$ & 0 & NS \\
\hline & $24^{\text {th }}$ day & 0 & $6.1 \%$ & NS \\
\hline \multirow[t]{3}{*}{ Anorexia } & $10^{\text {th }}$ day & $40.9 \%$ & $13.9 \%$ & $\mathrm{P}<0.05$ \\
\hline & $17^{\text {th }}$ day & $30 \%$ & $8.3 \%$ & $\mathrm{P}<0.05$ \\
\hline & $24^{\text {th }}$ day & $15 \%$ & $15.2 \%$ & NS \\
\hline
\end{tabular}

help preserve the gut flora. The beneficial changes in gut flora and environment by synbiotics administration may enforce systemic immune function and decrease the incidence of septic complications such as pneumonia, enteritis, and bacteremia in patients with severe systemic inflammatory response syndrome (SIRS). ${ }^{21} \mathrm{~A}$ common mistake is the belief that prebiotic can increase the population and/or function of probiotic, whereas prebiotics stimulate the flora that is already present. According to similar evidence, we chose this regimen in our study design. Study drug contained Lactobacillus casei, Lactobacillus rhamnosus, Streptococcus thermophilus, Bifidobacterium breve, Lactobacillus acidophilus, Bifidobacterium longum, Lactobacillus bulgaricus, and also Fructooligosaccharide as prebiotic, Magnesium, and a vegetable capsule (hydroxypropyl methyl cellulose).
In our study, ITT analysis showed that adding synbiotic to commercial anti $H$. pylori regimen led to a better drug tolerance. Therefore this group of patients uses their medications well. The eradication rate of $92.1 \%$ in this group is a reasonable rate. Cure rates below $80 \%$ by ITT analysis, are the accepted threshold separating acceptable from unacceptable treatment results. Grading clinical studies into effectiveness categories using prespecified criteria would allow clinicians to objectively identify and compare regimens. Graham and colleagues offer a therapy report card similar to that used to grade the performance of school children. The ITT cure rate categories are: "F" or unacceptable $(80 \%)$, "D" or poor (81-84\%), "C" or fair (85-89\%), "B" or good (90-95\%), and " $\mathrm{A}$ " or excellent (95-100\%). Regimens scoring as 
Table 4: Comparison of GDQ score in the two groups before and after treatment

\begin{tabular}{|c|c|c|c|c|c|}
\hline & \multicolumn{3}{|c|}{$(\mathrm{SD} \pm$ mean) GDQ score } & \multirow[b]{2}{*}{$95 \% \mathrm{CI}$} & \multirow[b]{2}{*}{ P value } \\
\hline & Before treatment & After treatment & Difference & & \\
\hline Placebo & $3.36 \pm 3.25$ & $1.76 \pm 2.61$ & $1.6 \pm 3.09$ & $0.15-3.04$ & $<.05$ \\
\hline Probiotic & $2.76 \pm 2.07$ & $1.53 \pm 1.54$ & $1.23 \pm 2.37$ & $0.39-2.07$ & $<.05$ \\
\hline
\end{tabular}

"B" or "good" can be used if "excellent" results are not obtainable. $^{22}$ As a general rule, clinicians should prescribe therapeutic regimens that have a $\geq 90 \%$ or, preferably, $\geq 95 \%$ eradication rate locally as mentioned by Rimbara and co-workers. ${ }^{23}$ They emphasized that if no available regimen can achieve a $\geq 90 \%$ eradication rate, clinicians should use the most effective regimen(s) available locally. ${ }^{23}$ This goal was achieved by our proposed regimen.

Gastroduodenal microbiota could protect the gut mucosa. Consequently the intake of exogenous lactic acid bacteria, particularly those with probiotic properties, may reinforce these protective functions in the stomach by maintaining local microbiological homeostasis, interfering with $H$. pylori and/or decreasing inflammatory processes. ${ }^{24}$

Two main types of substances have been implicated in the inhibition of $H$. pylori by lactic acid bacteria: SCFAs and bacteriocins. Probiotics produce SCFAs and have an important role in decreasing $\mathrm{pH}$. A dose-dependent inhibition of $H$. pylori growth has been observed with acetic and lactic acid, the later demonstrating the most intense effect. ${ }^{25}$ Such antimicrobial activity may be due to a direct effect on Helicobacter and also the inhibition of its urease activity. Bacteriocins are small, heat-resistant peptides, which are synthesized by several bacterial species including lactic acid bacteria with potential antiH. pylori activity. This peptide with other heat-stable proteinaceous compounds are capable of inhibiting the growth of both antibiotic-resistant and -sensitive strains of H. pylori. ${ }^{26-27}$

Accumulating evidence suggests an important role of interleukin-8 (IL-8) in H. pylori infection associated peptic ulcer and it is an important mediator in the immunopathogenesis of chronic gastritis caused by H. pylori. ${ }^{28}$

It has been demonstrated that cagA and vacA-s1 positive strains of $H$. pylori induce production of IL-8 in the gastric mucosa, both in vivo and in vitro. In addition, an association between the mucosal levels of IL-8 and severity of gastritis and presence of PUD has also been reported. ${ }^{29}$ IL- 8 appears paramount in the acute inflammatory response to $H$. pylori infection, as this gene is involved in all significant response pathways in the initial cellular response to infection. Several authors have demonstrated increase in IL-8 in response to $H$. pylori in both in vivo ${ }^{30}$ and in vitro studies. ${ }^{31-32}$ IL-8 is a key chemokine in accumulating neutrophils. Gastric mucosal IL-8 levels have shown a positive correlation with the degree of stomach corpus inflammation. ${ }^{33}$ Some probiotics exert an anti-inflammatory effect by decreasing dose dependently the release of IL-8, thus they prevent H. pylori colonization and the development of gastritis. Lactobacillus acidophilus (johnsonii) La1 decrease gastric inflammation in colonized animals. ${ }^{34}$

Increased levels of some growth factors and prostaglandins have been implicated in the protective effect of some strains of B. breve (that was present in our study drug) and B. bifidum against gastric ulcers induced by acetic acid or ethanol in rats. ${ }^{35}$

Other possible mechanisms of protection induced by probiotics including the stimulation of the expression of gastric mucins, decreases in bacterial overgrowth, stimulation of local immune responses and release of antioxidant substances. ${ }^{36}$

In our study, the most prominent benefit of synbiotics on drug side effects was improvement of anorexia. Among the various strains of probiotic, Lactobacillus causes some benefits. Increased appetite is one of them. There were four different Lactobacillus species in our study used product. In a recent meta-analysis the incidence of diarrhea was significantly reduced in the probiotic group ( $\mathrm{OR}=0.21,95 \% \mathrm{CI}$ : $0.06-0.74)$, whereas the incidence of taste disorders, metallic taste, vomiting, nausea, and epigastric pain did not differ significantly between the probiotic group and the control group. ${ }^{37}$ In another recent comprehensive analysis, at a first glance, 
probiotics seem effective in decreasing side effects. At a closer look, however, evidence only supports these claims for specific probiotic strains, ineffective antibiotic therapies, and low-quality trials. ${ }^{38}$

Previous meta-analyses and review articles have suggested that probiotic supplements could improve H. pylori eradication. ${ }^{37-38}$ Our study showed that probiotics with prebiotics could raise this rate significantly.

Probiotics are under valuable studies, as the idea holds promise for human health and well-being, and corresponding commercial opportunities. Protection of consumers requires health claims to be confirmed by important scientific documentations. Overall scientific demonstration of probiotic effects requires defining a healthy body microbiota and interactions between these microbiota and host factors, and the difficulty to define probiotic effectiveness in health and disease. Recent developments of high-throughput sequencing technology and the consequent progresses of metagenomics represent a new approach for the future of probiotics research. ${ }^{39}$

Our study had some limitations. First of all, a high dropout rate in conventional regimen was occurred. Clarithromycin was the main cause of treatment discontinuation. We could not obligate patients with intolerable side effects to continue the regimen despite their desire.

Cost is a major consideration in many countries such as ours. The cost of adding probiotic supplementations to standard regimens was a problem. Accompanying antibiotics with probiotics may decrease the number of effective bacteria in consumed capsules. This may be another difficulty of our work that could influence our results. On the other hand, the results may not be applicable to other countries and populations. The low rate of withdrawal in probiotic group may not be reproducible outside the trial setting, as the prescribing physician does not explain the temporary nature of drug side effects and the significance of completing the prescribed regimen. Finally, although adding probiotics causes an improvement over current therapies, it does not decrease the duration of therapy. Combined probiotic supplementation appears to be a promising therapeutic agent for treatment of some diseases in adults. However, there is concern that although probiotics are considered non-pathogenic, it may be infective when the patient is acutely ill or immunosuppressed. Some factors should be considered, including the safety, strain selection, product stability, and formulation. It should always be kept in mind that probiotic must stay viable to bring the beneficial effect and its viability is affected by temperature, humidity, and oxygen concentration. The product should also be delivered in right dose. Lower dose of probiotic in the market may not provide the beneficial effects as reported in clinical studies. Therefore, further controlled clinical studies, as well as good storage, handling and distribution is essential to explore the therapeutic benefit for possible practical clinical application. We recommend designing other studies in order to define the best duration and dose of such probiotic added combination regimens.

In the future, considerable advances will be made in understanding the evolution of the organism and pathogenesis of diseases. Although combination therapies have high rates of eradication, the preferred treatment regimen would be one which use less medications especially with a lower dose and with a short course with more tolerability or without any adverse effects.

Finally, we recommend adding synbiotic supplementations to various anti $H$. pylori regimens, for a better eradication in patients without any contraindications to their use.

\section{CONFLICT OF INTEREST}

The authors declare no conflict of interest related to this work.

\section{REFERENCES}

1. Dooley CP, Cohen H, Fitzgibbons PL, Bauer M, Appleman MD, Perez-Perez GI, et al. Prevalence of Helicobacter pylori infection and histologic gastritis in asymptomatic persons. N Engl J Med 1989;321:1562-6. doi: 10.1056/NEJM198912073212302

2. Kashiwagi H. Ulcers and gastritis. Endoscopy 2003;35:914. doi: $10.1055 / \mathrm{s}-2003-36397$

3. Plummer M, Franceschi S, Munoz N. Epidemiology of gastric cancer. IARC Sci Publ 2004:311-26.

4. Suerbaum S, Michetti P. Helicobacter pylori infection. $N$ Engl J Med 2002;347:1175-86. doi: 10.1056/NEJM$\mathrm{ra} 020542$

5. Vergara M, Vallve M, Gisbert JP, Calvet X. Meta-analysis: comparative efficacy of different proton-pump inhibitors in triple therapy for Helicobacter pylori eradica- 
tion. Aliment Pharmacol Ther 2003;18:647-54. doi: 10.1046/j.1365-2036.2003.01746.x

6. McColl KE. Clinical practice. Helicobacter pylori infection. $N$ Engl J Med 2010;362:1597-604. doi: 10.1056/ NEJMcp 1001110.

7. Fischbach L, Evans EL. Meta-analysis: the effect of antibiotic resistance status on the efficacy of triple and quadruple first-line therapies for Helicobacter pylori. Aliment Pharmacol Ther 2007;26:343-57. doi:10.1111/j.13652036.2007.03386.x

8. Myllyluoma E, Veijola L, Ahlroos T, Tynkkynen S, Kankuri E, Vapaatalo H, et al. Probiotic supplementation improves tolerance to Helicobacter pylori eradication therapy--a placebo-controlled, double-blind randomized pilot study. Aliment Pharmacol Ther 2005;21:1263-72. doi: 10.1111/j.1365-2036.2005.02448.x

9. Isolauri E, Kirjavainen PV, Salminen S. Probiotics: a role in the treatment of intestinal infection and inflammation? Gut 2002;50:III54-9. doi:10.1136/gut.50.suppl_3.iii54

10. Marteau P, Seksik P, Jian R. Probiotics and intestinal health effects: a clinical perspective. $B r J$ Nutr 2002;88:S51-7. doi:10.1079/BJN2002629

11. Cindoruk M, Erkan G, Karakan T, Dursun A, Unal S. Efficacy and safety of Saccharomyces boulardii in the 14-day triple anti-Helicobacter pylori therapy: a prospective randomized placebo-controlled double-blind study. Helicobacter 2007;12:309-16. doi: 10.1111/j.1523-5378.2007.00516.x

12. Song MJ, Park DI, Park JH, Kim HJ, Cho YK, Sohn CI, et al. The effect of probiotics and mucoprotective agents on PPI-based triple therapy for eradication of Helicobacter pylori. Helicobacter 2010;15:206-13. doi: 10.1111/j.1523-5378.2010.00751.x

13. Franceschi F, Cazzato A, Nista EC, Scarpellini E, Roccarina D, Gigante G, et al. Role of probiotics in patients with Helicobacter pylori infection. Helicobacter 2007; 2:59-63. doi: 10.1111/j.1523-5378.2007.00565.x

14. Aiba Y, Suzuki N, Kabir AM, Takagi A, Koga Y. Lactic acid-mediated suppression of Helicobacter pylori by the oral administration of Lactobacillus salivarius as a probiotic in a gnotobiotic murine model. Am J Gastroenterol 1998;93:2097-101. doi:10.1111/j.1572-0241.1998.00600.x

15. Pinchuk IV, Bressollier P, Verneuil B, Fenet B, Sorokulova IB, Megraud F, et al. In vitro anti-Helicobacter pylori activity of the probiotic strain Bacillus subtilis 3 is due to secretion of antibiotics. Antimicrob Agents Chemother 2001;45:3156-61. doi: 10.1128/AAC.45.11.31563161.2001

16. Smith AD, Gillen D, Cochran KM, El-Omar E, McColl KE. Dyspepsia on withdrawal of ranitidine in previously asymptomatic volunteers. Am J Gastroenterol 1999;94:1209-13. doi:10.1111/j.1572-0241.1999.01068.x

17. de Boer WA, Thys JC, Borody TJ, Graham DY, O’Morain C, Tytgat GN. Proposal for use of a standard side effect scoring system in studies exploring Helicobacter pylori treatment regimens. Eur J Gastroenterol Hepatol 1996;8:641-3.

18. Report of a Joint FAO/WHO Expert Consultation on
Evaluation of Health and Nutritional Properties of Probiotics in Food Including Powder Milk with Live Lactic Acid Bacteria. FAO FOOD AND NUTRITION PAPER 2001;85.

19. Gibson GR, Roberfroid MB. Dietary modulation of the human colonic microbiota: introducing the concept of prebiotics. J Nutr 1995;125:1401-12.

20. Shimizu K, Ogura H, Goto M, Asahara T, Nomoto K, Morotomi M, et al. Synbiotics decrease the incidence of septic complications in patients with severe SIRS: a preliminary report. Dig Dis Sci 2009;54:1071-8. doi: 10.1007/s10620-008-0460-2.

21. Shimizu K, Ogura H, Asahara T, Nomoto K, Morotomi M, Tasaki O, et al. Probiotic/synbiotic therapy for treating critically ill patients from a gut microbiota perspective. Dig Dis Sci 2013;58:23-32. doi: 10.1007/s10620-012-2334-x.

22. Graham DY, Lu H, Yamaoka Y. A report card to grade Helicobacter pylori therapy. Helicobacter 2007;12:2758. doi: 10.1111/j.1523-5378.2007.00518.x

23. Rimbara E, Fischbach LA, Graham DY. Optimal therapy for Helicobacter pylori infections. Nat Rev Gastroenterol Hepatol 2011;8:79-88. doi: 10.1038/nrgastro.2010.210.

24. Alsahli M, Michetti P. Lactobacilli for the management of Helicobacter pylori. Nutrition 2001;17:268-9. doi:10.1016/S0899-9007(00)00475-5

25. Midolo PD, Lambert JR, Hull R, Luo F, Grayson ML. In vitro inhibition of Helicobacter pylori NCTC 11637 by organic acids and lactic acid bacteria. J Appl Bacteriol 1995;79:475-9. doi: 10.1111/j.1365-2672.1995.tb03164.x

26. Collado MC, Gonzalez A, Gonzalez R, Hernandez M, Ferrus MA, Sanz Y. Antimicrobial peptides are among the antagonistic metabolites produced by Bifidobacterium against Helicobacter pylori. Int J Antimicrob Agents 2005;25:385-91. doi: 10.1016/j.jjantimicag.2005.01.017

27. Tsai CC, Huang LF, Lin CC, Tsen HY. Antagonistic activity against Helicobacter pylori infection in vitro by a strain of Enterococcus faecium TM39. Int J Food Microbiol 2004;96:1-12. doi:10.1016/j.ijfoodmicro.2003.10.019

28. Hisatsune J, Nakayama M, Isomoto H, Kurazono H, Mukaida N, Mukhopadhyay AK, et al. Molecular characterization of Helicobacter pylori VacA induction of IL-8 in U937 cells reveals a prominent role for p38MAPK in activating transcription factor-2, cAMP response element binding protein, and NF-kappaB activation. J Immunol 2008;180:5017-27. doi: 10.4049/jimmunol.180.7.5017

29. Bartchewsky W Jr, Martini MR, Masiero M, Squassoni AC, Alvarez MC, Ladeira MS,, et al. Effect of Helicobacter pylori infection on IL-8, IL-1beta and COX-2 expression in patients with chronic gastritis and gastric cancer. Scand J Gastroenterol 2009;44:153-61. doi: $10.1080 / 00365520802530853$.

30. Naito Y, Ito M, Watanabe T, Suzuki H. Biomarkers in patients with gastric inflammation: a systematic review. $\mathrm{Di}$ gestion 2005;72:164-80. doi:10.1159/000088396

31. Kim SY, Lee YC, Kim HK, Blaser MJ. Helicobacter pylori CagA transfection of gastric epithelial cells induces interleukin-8. Cell Microbiol 2006;8:97-106. 
doi: 10.1111/j.1462-5822.2005.00603.x

32. Sharma SA, Tummuru MK, Miller GG, Blaser MJ. Interleukin-8 response of gastric epithelial cell lines to Helicobacter pylori stimulation in vitro. Infect Immun 1995;63:1681-87.

33. Xuan J, Deguchi R, Yanagi H, Ozawa H, Urano T, Ogawa $\mathrm{Y}$, et al. Relationship between gastric mucosal IL-8 levels and histological gastritis in patients with Helicobacter pylori infection. Tokai J Exp Clin Med 2005;30:83-8.

34. Michetti P, Dorta G, Wiesel PH, Brassart D, Verdu E, Herranz M, et al. Effect of whey-based culture supernatant of Lactobacillus acidophilus (johnsonii) La1 on Helicobacter pylori infection in humans. Digestion 1999;60:203-9. doi:10.1159/000007660

35. Nagaoka M, Hashimoto S, Watanabe T, Yokokura T, Mori Y. Anti-ulcer effects of lactic acid bacteria and their cell wall polysaccharides. Biol Pharm Bull 1994;17:1012-17. doi.org/10.1248/bpb.17.1012

36. Gotteland M, Brunser O, Cruchet S. Systematic review: are probiotics useful in controlling gastric colonization by Helicobacter pylori? Aliment Pharmacol Ther 2006;23:1077-86. doi: 10.1111/j.13652036.2006.02868.x

37. Rong Zhu, Kan Chen, Yuan-Yuan Zheng, Hua-Wei Zhang, Jun-Shan Wang, Yu-Jing Xia,et al. Meta-analysis of the efficacy of probiotics in Helicobacter pylori eradication therapy. World J Gastroenterol 2014;20: 18013 21. doi: 10.3748/wjg.v20.i47.18013.

38. Dang Y, Reinhardt JD, Zhou X, Zhang G. The effect of probiotics supplementation on Helicobacter pylori eradication rates and side effects during eradication therapy: a meta-analysis. PLoS One 2014;9:e111030.

doi: 10.1371/journal.pone.0111030. eCollection 2014.

39. Gueimonde M, Collado MC. Metagenomics and probiotics. Clin Microbiol Infect 2012;4:32-4. doi: $10.1111 / j .1469-0691.2012 .03873 . x$. 